\title{
APPROXIMATION BY QUOTIENTS OF RATIONAL INNER FUNCTIONS
}

\author{
JOHN N. MCDONALD
}

\begin{abstract}
Let $u$ be a continuous unimodular function on the $n$-dimensional torus $T^{n}$. It is shown that $u$ can be uniformly approximated by quotients of rational inner functions.
\end{abstract}

Let $T$ denote the unit circle. Let $u$ be a Lebesgue measurable function on $T$ such that $|u|=1$ a.e. In [1] Douglas and Rudin proved that: given $\varepsilon>0$, there exist inner functions $g_{1}$ and $g_{2}$ such that $\left\|u-g_{1} \bar{g}_{2}\right\|<\varepsilon$. $(\|\cdot\|$ indicates the essential-sup norm.) In this paper we establish a continuous analogue of Douglas and Rudin's result on the torus $T^{n}$. We show that, if $v$ is a continuous unimodular function on $T^{n}$, then $v$ can be uniformly approximated by quotients of rational inner functions.

Let $I$ and $I_{+}$denote, respectively, the set of integers and the set of nonnegative integers. Let $\alpha=(a, b, \ldots, x) \in I^{n}$. We will use $z^{\alpha}$ to denote the function defined on $T^{n}$ by $z^{\alpha}\left(\xi_{1}, \xi_{2} \cdots \xi_{n}\right)=\xi_{1}^{a} \xi_{2}^{b} \cdots \xi_{n}^{x}$. A finite linear combination of the $z^{\alpha}$, where the $\alpha$ 's are taken from $I_{+}^{n}$, will be called a polynomial. If $p=\Sigma c(\alpha) z^{\alpha}$ is a polynomial, we will use the notation $\alpha(p)$ to denote the $n$-tuple $\left(\alpha_{1}(p), \ldots, \alpha_{n}(p)\right)$, where $\alpha_{i}(p)$ denotes the maximum $i$ th component of any $\alpha$ satisfying $c(\alpha) \neq 0$, and we will use $\tilde{p}$ to indicate the polynomial $\overline{\Sigma(\alpha)} z^{(\alpha(p)-\alpha)}$. Clearly, polynomials are also well defined over the closure of the open unit polydisk $D^{n}$. A rational inner function on $T^{n}$ is a function of the form $c z^{\alpha}(\tilde{p} / p)$, where $c$ is a constant with $|c|=1$, where $\alpha \in I_{+}^{n}$, and where $p$ is a polynomial having no zeros on the closure of $D^{n}$. (Our definition of rational inner function is essentially the same as the one in Rudin's book [3, p. 110].) Finally, let $U$ and $U_{0}$ denote, respectively, the set of continuous unimodular functions on $T^{n}$ and the set of continuous unimodular functions on $T^{n}$ having continuous logarithms. Note that $U$ and $U_{0}$ are both groups under the usual operation of (pointwise) multiplication of complex-valued functions.

Proposition. For each $u \in U$, there is an $\alpha \in I^{n}$ such that $z^{\alpha} u \in U_{0}$.

Proof. It can be shown that the factor group $U / U_{0}$ is isomorphic to the first Čech cohomology group $H^{1}$ of $T^{n}$, where the coefficients are taken from I. (This can be done quickly by applying the Arens-Royden theorem; see [2].)

Received by the editors June 20, 1978.

AMS (MOS) subject classifications (1970). Primary 41A20.

Key words and phrases. Rational inner function, polydisk.

() 1979 American Mathematical Society 0002-9939/79/0000-0218/\$01.50 
It is an exercise in algebraic topology to show that $H^{1}$ is isomorphic to $I^{n}$. Since $\left\{z^{\alpha} U_{0} \mid \alpha \in I^{n}\right\}$ is a free abelian group having $n$ generators, it follows that $U / U_{0}=\left\{z^{\alpha} U_{0} \mid \alpha \in I^{n}\right\}$.

THEOREM. Let $V$ denote the closure in the topology of uniform convergence of functions in $U$ of the form $g \bar{g}_{1}$, where $g$ and $g_{1}$ are rational inner functions. Then $V=U$.

Proof. Since $V$ is a subgroup of $U$ and since $z^{\alpha} \in V$ for every $\alpha \in I^{n}$, it follows from the proposition above that $U_{0} \subseteq V$ implies $V=U$. Suppose $u \in U_{0}$, then $u=e^{i f}$, where $f$ is a real-valued continuous function on $T^{n}$. Hence, for each positive integer $m$, we have $u=\left(u_{m}\right)^{m}$, where $u_{m}=e^{i f / m}$. By choosing $m$ sufficiently large, the real part of $u_{m}$ can be made uniformly close to 1 . It follows that, in order to show that $U_{0} \subseteq V$, it suffices to prove that every $u \in U_{0}$ of the form $u=(v)^{2}$, where $\operatorname{Re} v \geqslant \frac{1}{2}$, lies in $V$. Let $\varepsilon>0$ be given. By the Stone-Weierstrass theorem there exists a polynomial $p$ and an $\alpha \in I_{+}^{n}$ such that $\left\|v-\bar{z}^{\alpha} p\right\|<\varepsilon / 3,\left\|(1 / v)-1 /\left(\bar{z}^{\alpha} p\right)\right\|<\varepsilon / 3,\left\|\bar{z}^{\alpha} p\right\|^{-1}<2$, and $\frac{1}{4}<\operatorname{Re} \bar{z}^{\alpha} p$. It follows that

$$
\left\|\bar{v}^{2}-\left(z^{\alpha} \bar{p}\right) /\left(\bar{z}^{\alpha} p\right)\right\|<\left\|\bar{v}^{2}-\bar{v} /\left(\bar{z}^{\alpha} p\right)\right\|+\left\|\bar{v} /\left(\bar{z}^{\alpha} p\right)-\left(z^{\alpha} \bar{p}\right) /\left(\bar{z}^{\alpha} p\right)\right\|<\varepsilon .
$$

Note that $\bar{p}=\bar{z}^{\alpha(p)} \tilde{p}$ on $T^{n}$. Hence, $\left(z^{\alpha} \tilde{p}\right) /\left(\bar{z}^{\alpha} p\right)=z^{2 \alpha-\alpha(p)} \tilde{p} / p$. Thus, the proof will be completed if we can show that $p$ has no zeros in the closure of $D^{n}$. Note that the function $\operatorname{Re} \bar{z}^{\alpha} p$ is well defined on the closure of $D^{n}$ and is harmonic in each variable on $D^{n}$. It follows from the minimum principle for harmonic functions that $\operatorname{Re} \bar{z}^{\alpha} p \geqslant \frac{1}{4}$ on the closure of $D^{n}$. In particular $p$ cannot have a zero on the closure of $D^{n}$.

\section{REFERENCES}

1. R. G. Douglas and Walter Rudin, Approximation by inner functions, Pacific J. Math. 31 (1969), 313-320.

2. T. W. Gamelin, Uniform algebras, Prentice-Hall, Englewood Cliffs, N. J., 1969.

3. W. Rudin, Function theory on polydisks, Benjamin, New York, 1969.

Department of Mathematics, Arizona State University, Tempe, Arizona 85281 